\title{
Investigation of 2-GHz Threshold Levels for Radio Waves in the NIGER Delta Region, CRS, Nigeria
}

\author{
Okon, Bernard Edem \\ ${ }^{1}$ Department of Physics, University of Calabar, Calabar, Cross River State, Nigeria
}

\begin{abstract}
Measurements and analyses of Radio wave transmission are basic necessities for optimization and planning of radio communication networks. These evaluations are usually done through series of propagation measurements, computation of intensive radio waves simulation, or by empirically estimating transmitted field strengths. In collaboration with M-TEL, Nigeria, measurements on W-CDMA based mobile networks, modeled IS-95 and cdma2000, were carried out. The study centered on 2G and 3G bands. The received signal was mixed and amplified to an in-phase quadrature baseband signal levels through a common DSP board for 900MHz and $2000 \mathrm{MHz}$. The recorded time interval for impulse response of data was $0.1 \mathrm{~s}$, while the mobile recording instrument maintained a low continuous velocity of approximately $1.5 \mathrm{~m} / \mathrm{s}$, along the trajectory leading to the specified locations. Samples from the results of the study indicate that: Calabar urban recorded mean signal path loss of 82.9dB, as against a predicted path loss of 94.5dB. Values for path loss mean square error and standard deviation were $4.48 \mathrm{~dB}$ and $4.48 \mathrm{~dB}$ respectively. Characteristically, the urban city has a flat surface and high concentration of buildings. Similarly, measured signal penetration recorded 5.9dB, as against predicted urban environmental path loss model of $2.7 \mathrm{~dB}-3.5 \mathrm{~dB}$. The remarkable difference between measured and predicted values for Calabar urban was attributed to obstructions of the signal path due to buildings. Results from the study can be used for validation of existing propagation models for urban, residential and office blocks located in plantations in the Niger Delta region.
\end{abstract}

Keywords: Attenuation coefficient, predicted path loss, propagation models, signal path loss.

\section{Introduction}

The establishment of effective radio communication system is usually encumbered with problems associated with complications arising from the transmission and reception of signal. Therefore, the design of effective and efficient wireless radio systems requires insight of the radio transmission characteristics and pathway. These parameters vary greatly with the operating frequency and the propagation environment.

In considering these parameters, individual links can be utilized by plethora of Base transceiver stations, when the distance between the up converter and down converter is sufficiently wide.

The height of the antenna at the mobile terminal is more often than not extremely small: normally below a small number of meters. Consequently, the receiver's antenna contains exceptionally small "clearance"; thus, permitting blockages and surface reflection in the neighborhood of the receiver [3]. This reduces the equipment's significant control of the propagation characteristics of the radio waves. Besides, these characteristics may have a propensity to vary from one place to the other if the mobile unit is in motion. Hence, the path link between the transmitter and the receiver can diverge from line-of-sight to one that is rigorously blocked by intervening buildings, traffic, foliage, terrain, etc.

Series of studies have been conducted on the transmission of cellular mobile at frequency range of $900 \mathrm{MHZ}$ and 1900 MHZ. Some of these studies related to penetration into urban office buildings and their vicinity. However, this article is an extract from a comprehensive study carried out on radio waves transmission characteristics between urban, suburban and rural residential locations in Cross River State.

\section{Theoretical Review}

A radio wave is a form of EM wave consisting of electric field $(€)$ and magnetic field $(\mathrm{H})$ components. It propagates through the free space with the speed of light (approximately, $3 * 10^{8} \mathrm{~m} / \mathrm{s}$ ). Free space refers to a medium where there is no interference or obstacle to the normal radiation and propagation of radio waves.

The electric field intensity (E) and magnetic field intensity $(\mathrm{H})$ of a wave are not independent. In plane waves, for example, the $\mathrm{E}$ and $\mathrm{H}$ vectors are mutually perpendicular and are related by the expression [2].

$$
\overline{\mathrm{E}}=\Gamma * \overline{\mathrm{H}}
$$

Where $\overline{\mathrm{E}}$, known as the pointing vector, is the power flux per unit area. $\bar{E}$, is always perpendicular to the plane of $\Gamma$ and $\overline{\mathrm{H}}$. Hence, in a plane wave the vector $\overline{\mathrm{E}}$ and $\overline{\mathrm{H}}$ are mutually perpendicular.

\subsection{Radio (EM) Waves in Free Space}

In free space, radio waves radiate uniformly in all directions from a point source. This is known as isotropic radiation. According to the inverse square law, the power density, $\mathrm{p}_{\mathrm{d}}$ is inversely proportional to the square of the distance, $r$, from the source [8].

$$
P_{d}=\frac{p_{t}}{4 \pi r^{2}}
$$

Where, $\mathrm{p}_{\mathrm{t}}$ is the transmitting power at the source. If $\mathrm{r}$ is relatively greater than the wavelength of the transmitted signal, then the wave is in a plane wave. In such a case, E and $\mathrm{H}$ are related by the formula.

$$
E=Z H
$$




\section{International Journal of Science and Research (IJSR) \\ ISSN (Online): 2319-7064 \\ Index Copernicus Value (2013): 6.14 | Impact Factor (2014): 5.611}

Where $\mathrm{Z}$ is known as the characteristic impedance of the medium, and, $\mathrm{E}$ and $\mathrm{H}$ are both rms values. $\mathrm{Z}$ is given by the expression.

$$
Z=\sqrt{\frac{\mu}{\varepsilon}}
$$

Where $=$ permeability of the medium, and $\varepsilon$ permittivity of the medium.

In free space,

$$
\begin{aligned}
& \mu=\mu_{o}=4 \pi \times 10^{-7} \text { Henry /metre } \\
& \varepsilon=\varepsilon_{0}=8.854 \times 10^{-12} \text { Farads } / \text { metre }
\end{aligned}
$$

Hence, the characteristics impedance of free space is

$$
Z_{o}=\sqrt{\frac{\mu_{o}}{\varepsilon_{o}}}=\sqrt{\frac{4 \pi \times 10^{-7}}{8.854 \times 10^{-12}}}=120 \pi \mathrm{ohm}
$$

The electric field intensity at a point $r$ meters away from the point source is:

$$
E=\sqrt{P_{d} \cdot Z_{o}}=\sqrt{\frac{P_{t} \cdot 120 \pi}{4 \pi r^{2}}}=\sqrt{\frac{30 P_{t}}{r^{2}}}
$$

This means that $E$ is inversely proportional to $\mathrm{r}$, while $P_{d}$ is inversely proportional to $\mathbf{r}^{2}[8]$.

\subsubsection{Properties of Radio Waves Propagation.}

\section{(a) Polarization}

Polarization refers to the physical orientation of the radiated electromagnetic waves in space. A wave can be vertically, horizontally, or elliptically polarized. The direction of the E vector normally designates the polarization of the wave. Thus, a vertically polarized wave implies that the $E$ vector of the wave is in the vertical direction [2]

It is very imperative to understand the polarization of a transmitted wave with the intention of employing a similar polarized antenna at the receiving end.

Voltage of such antenna can be measured with a measurement receiver connected to the antenna. When the ,antenna factore is known, and the antenna voltage is measured, the corresponding field strength can be calculated as [7]:

$$
E=K^{*} U
$$

Where:

$\mathrm{E}=$ Electric field strength $[\mathrm{V} / \mathrm{m}]$

$\mathrm{K}=$ Antenna factor $[1 / \mathrm{m}]$

$\mathrm{U}=$ Antenna voltage $[\mathrm{V}]$

Similarly, when the free space antenna gain is known, the antenna factor can also be calculated.

\section{(c) Tropospheric Scattering}

The troposphere is that part of the atmosphere extending up to a height of $10-15 \mathrm{~km}$ above the surface of the earth [3]. In normal atmospheric conditions, the refractive index decreases uniformly with increase height. However, with different meteorological conditions, a phenomenon known as "temperature inversion" occurs at the lower part of the atmosphere. Under this conditions temperature increases with height; thus, leading to a rapid increase in humidity with height. These irregularities in the refractive index may alter incident radio wave by certain degree. Therefore, if two directional antennas are pointed so that their beams intersect the troposphere within the temperature-inversion region, then communication line can be established between the two antennas due to scattering. Troposphere scattering is a very reliable means of communication above a certain horizon at the UHF level. It is also used as an alternative to microwave (LOS) links over difficult terrains. Path links ranging from $300-500 \mathrm{Km}$ distance can be so established [10]. The mean path loss can also be written as the quotient of the transmitted power to the received powers:

$$
\mathrm{L}[\mathrm{dB}]=10 \log 10
$$

Where Tx and Rx denote the transmitted and the average received power in Watts [W]

\section{Measurements Specification and Procedures}

In collaboration with M-TEL, South East Zonal Deputy General Manager, wideband (W-CDMA) pilot scanner and a laptop for carrying out measurements on W-CDMA based mobile networks, (modeled IS-95 and cdma2000) were accessed for this study. The scanner has two radio units that covered both second and third generation (2G and 3G) mobile networks. Additional measurement equipment were the omni-directional antenna, a weather station, a global positioning system (GPS) unit and a laptop running a software capable of controlling and logging results from the measurement. The measurement system can track up to eight channel (pilot) signals concurrently, and the corresponding peak (correlation peak) pilot signal; thereby allowing for tracking of speedy signal fades.

The W-CDMA scanner consists of a number of independent hardware modules, with major internal components such as:

- Digital signal processing (DSP) board, incorporating a field programmable gate array (FPGA) and various analog and digital inputs and outputs converters.

- Co-processor, supplementing the DSP with high-level data processing and a universal serial bus (USB) interface to an external computing laptop.

- $900 \mathrm{MHz}$ and $2000 \mathrm{MHz}$ radios designed to receive signals from 2G and3G CDMA systems.

- GPS receiver obtaining spatial position information during measurements.

In order to measure both $2 \mathrm{G}$ and $3 \mathrm{G}$ bands, two independent radios were incorporated. The received signal was mixed and amplified to an in-phase quadrature baseband signal. The DSP board was used common to both $900 \mathrm{MHz}$ radio and $2000 \mathrm{MHz}$

Other supporting components were: Radio Analog (MUX) control unit, sensor data control, sensor data weather station, In-phase Quadrature Main DSP board, High speed serial link, (25Mbps) DSP Co-processor, GPS receiver, USB link to Laptop(PC), W-CDMA scanner integrated hardware components and interactions radios. Also, there were two high-speed analog-to-digital (A/D) converter channels. Since the scanner is limited to $2 \mathrm{G}$ and $3 \mathrm{G}$ operation, the $900 \mathrm{MHz}$ and $2000 \mathrm{MHz}$ radio signals were multiplexed together, while 


\section{International Journal of Science and Research (IJSR) \\ ISSN (Online): 2319-7064}

Index Copernicus Value (2013): 6.14 | Impact Factor (2014): 5.611

the scanner operates in either $2 \mathrm{G}$ or $3 \mathrm{G}$ mode at one time, depending on the configuration. The baseband signals were processed by both FPGA-based signal processing hardware and DSP software, to correlate the received spread-spectrum signals. Thus, detecting the pilot and synchronization (sync) channel signals. The pilot andsync data were passed to a second DSP co-processor through serial link. The secondary processor was responsible for formatting and transferring the pilot and sync data to the measurement laptop. In addition, the co-processor managed the GPS receiver.

The main operator interface is the graphic user interface (GUI) software running on a laptop. The scanner GUI allows the operator to input control commands and to display the pilot. Control commands may be passed from the scanner GUI to the co-processor and when necessary to the main DSP. The radio hardware is also equipped with associated interfacing requirements to the main DSP board, Antenna 's Low noise amplifiers, RF Switch Band pass filter, $880 \pm$ $12.5 \mathrm{MHz}, 900 \mathrm{MHz}$ radio, Frequency control temperature, Sampling clock, In-phase Quadrature, RSSI (16-bit A/D), VCXO (16-bit D/A), etc.

\subsection{Network Hardware Configuration}

The system was configured to operate under $900 \mathrm{MHz}$ through the following procedures.

- The RF signal input to the radio was obtained through the antenna, which was selected via transistor-transistor logic (TTL) signal output from the DSP. The main function of the radio was to amplify the input RF signal using suitable automatic gain control (AGC) mechanism.

- The radio also incorporated a frequency synthesizer, which was programmed via a serial line from the digital module. Thereby, allowing for the definition of the CDMA RF channels.

- It is equipped with a voltage control crystal oscillator (VCXO), which is the source of the clock frequencies used by the radio.

- The radio AGC outputs a receiver signal strength indicator (RSSI) signal, which covered the receiver dynamic range of -105 to $-30 \mathrm{dBm}$.

A laptop running through GUI controlled all the scanner features and enables selected parameters to be logged to fileenabled for later off-line data analysis. In the upper toolbar, there were buttons that were used to control the measurements in real time, or when playing back measured data (run, stop, recorded.). Two check- boxes used to activate or deactivate the GPS unit and the weather station, were positioned in the toolbar's upper right area. The radio antenna is shown in Fig. 1.

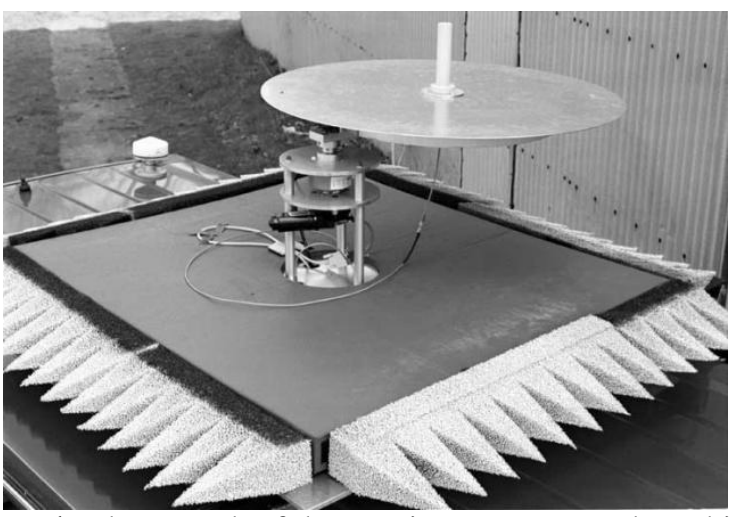

Figure 1: Photograph of the rotating antenna on the vehicleroof

\subsection{Implementation}

The radio transmission path profile measurements started at OkoiArikpo House, a-multi story building located in the heart of Calabar (urban) as shown in Fig. 2.

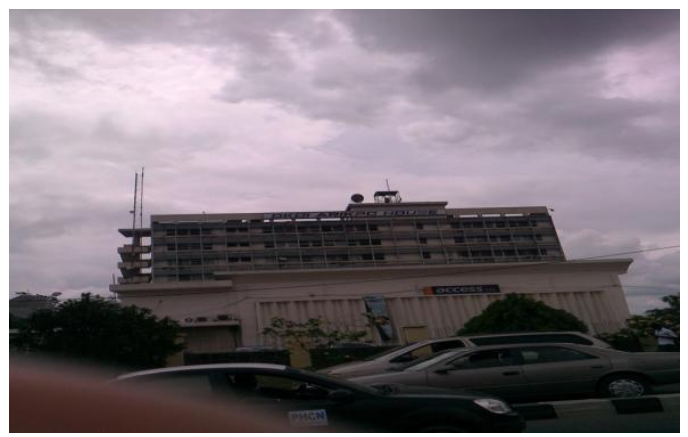

Figure 2: Measurement location with the transmission point on the rooftop of the OkoiArikpo building, Calabar.

To carry out measurements, the decoder's antenna employed was Omni-directional, vertically polarized and stationed permanently on the top of a motor vehicle at a height of $2.4 \mathrm{~m}$ beyond ground level. Also, at the top of OkoiArikpo House in Calabar (Fig.2), the transmitting aerial was installed omnidirectional, $12-\mathrm{dBi}$, with a beam width of $3 \mathrm{~dB}$, and $6^{0}$ polarizations. The antenna was installed on top of the building under consideration in Calabar, Cross River State, Nigeria at a height of 6.5 meter. During the measurements, the recorded time interval for impulse response of data was $0.1 \mathrm{~s}$, whereasthe automobile was accelerating at a low continuous velocity of approximately, $1.5 \mathrm{~m} / \mathrm{s}$ along the road leading to the specified locations. Other rural and sub-urban locations selected for the study were Mfamosing, Akpabio, Odukpani and Akamkpa. On each route under investigation, ten designations were selected to examine the terrain, the signal path loss and topography. These made the total measurement points to be fifty. At the terminal point of each measurement route, a no-configured radio-return-journey was taken back to the transmitting point (OkoiArikpo House), from where a new trip commenced for the next route. The receiver module was again configured and activated to take the next measurement. Similar measurements were also carried out inside the buildings with the transmitter at one side and the receiver at adjacent side. 


\section{International Journal of Science and Research (IJSR) \\ ISSN (Online): 2319-7064}

Index Copernicus Value (2013): 6.14 | Impact Factor (2014): 5.611

\section{Results and Discussion}

The result is based on extensive sets of measurements data that can be used for validation of existing propagation models for residential and office blocks located in plantations. Table 1 to Table 5 are measured mean values of results obtained on signal strength and transmission loss.

In order to perform a meaningful comparison between the measured values and the predicted values, we have to Consider the uncertainties involved in a particular environment. Both the measured and the predicted values were not exact; they consist of nominal values with varying uncertainty ranges. Since both measurements and prediction path loss analyses yielded different values even when they described the same event, a common method to express the mismatch between these two distributions could be the Wasserstein Metric [8]. However, in this case, attention was more on the figure of mismatch between the two measured and the predicted values. Besides, to quantify the accuracy of our propagation measurements, the empirical values were compared with earlier works done through measurements under similar environment. These values were further tested by calculating the standard-deviation (std. dev.) and the rootmean-square error (mse) between accessible figures (predicted or empirical) and measurement data reported in another publication.

Table 1: Measured Transmission Characteristics for buildings at Akamkpa urban.

\begin{tabular}{|c|c|c|}
\hline Building & Empirical $\alpha_{b}(\mathrm{~dB} / \mathrm{m})$ & Range of $L_{t}(\mathrm{~dB})$ \\
\hline 1 & 1.97 & $16-27$ \\
\hline 2 & 2.10 & $17-30$ \\
\hline 3 & 2.24 & $15-34$ \\
\hline 4 & 2.69 & $21-28$ \\
\hline 5 & 3.16 & $14-34$ \\
\hline Average & 2.43 & $17-31$ \\
\hline
\end{tabular}

Table 2: Measured transmission characteristics for buildings at Ugep urban.

\begin{tabular}{|c|c|c|}
\hline Building & Empirical $\alpha_{b}(\mathrm{~dB} / \mathrm{m})$ & Range of $\mathrm{L}_{t}(\mathrm{~dB})$ \\
\hline 1 & 1.86 & $15-30$ \\
\hline 2 & 1.86 & $14-34$ \\
\hline 3 & 1.57 & $30-49$ \\
\hline 4 & 2.02 & $22-45$ \\
\hline 5 & 1.72 & $28-47$ \\
\hline Average & 1.88 & $22-41$ \\
\hline
\end{tabular}

Table 3: Measured transmission characteristics for buildings at Ikom urban.

\begin{tabular}{|c|c|c|}
\hline Building & Empirical $\alpha_{b}(\mathrm{~dB} / \mathrm{m})$ & Range of $L_{t}(\mathrm{~dB})$ \\
\hline 1 & 2.06 & $16-29$ \\
\hline 2 & 2.06 & $21-34$ \\
\hline 3 & 1.77 & $20-25$ \\
\hline 4 & 2.03 & $23-32$ \\
\hline 5 & 2.40 & $22-45$ \\
\hline Average & 1.99 & $21-32$ \\
\hline
\end{tabular}

Table 4: Measured transmission characteristics for buildings at Calabar urban.

\begin{tabular}{|c|c|c|}
\hline Building & Empirical $\alpha_{b}(\mathrm{~dB} / \mathrm{m})$ & Range of $L_{t}(\mathrm{~dB})$ \\
\hline 1 & 2.02 & $26-33$ \\
\hline 2 & 2.37 & $31-41$ \\
\hline 3 & 3.80 & $51-64$ \\
\hline 4 & 1.38 & $14-30$ \\
\hline 5 & 1.97 & $22-35$ \\
\hline 6 & 2.00 & $24-40$ \\
\hline 7 & 1.58 & $22-45$ \\
\hline Average & 2.15 & $24-41$ \\
\hline
\end{tabular}

Table 5: Compressed Attenuation Coefficients for examined locations

\begin{tabular}{|c|c|c|c|c|c|}
\hline Buildings & Cat. & \multicolumn{3}{|c|}{ External dimensions } & \multicolumn{2}{|c|}{$\begin{array}{c}\text { Attenuation } \\
\text { coefficients } \alpha_{b}\end{array}$} \\
\hline & & $\begin{array}{c}\text { Dist. } \\
\text { (m) }\end{array}$ & $\begin{array}{c}\text { Width } \\
\text { (w) }\end{array}$ & $\begin{array}{c}\text { Height } \\
\text { (m) }\end{array}$ & $\mathrm{dB} / \mathrm{m}$ \\
\hline $\begin{array}{c}1^{*} \\
\text { (Akam.) }\end{array}$ & 1 & 73 & 9 & 9 & 2.24 \\
\hline $\begin{array}{c}2^{*} \\
\text { (Ugep) }\end{array}$ & 2 & 58 & 15 & 10 & 2.02 \\
\hline $3^{*}$ & 3 & 36 & 12 & 9 & 2.03 \\
(Ikom) & 3 & 40 & 14 & 23 & 1.38 \\
\hline $\begin{array}{c}4^{*} \\
\text { (Cal.) }\end{array}$ & 4 & 102 & 18 & 15 & 1.58 \\
\hline
\end{tabular}

From the results of the study, the Calabar urban signal path loss values recorded measured mean signal path loss of $82.9 \mathrm{~dB}$, against a predicted path loss of $94.5 \mathrm{~dB}$. In the analysis, the value for path loss mean square error was 4.48dB, and that for standard deviation was $4.48 \mathrm{~dB}$. Characteristically, the urban city has a flat surface and high concentration of buildings.

Similarly, measured signal penetration recorded $5.9 \mathrm{~dB}$, as against predicted urban environmental path loss exponent range of $2.7-3.5 \mathrm{~dB}$. The remarkable difference between measured and predicted values for Calabar urban is attributed to obstructions of the signal path due to buildings. Mfamosing is purely a suburb area with scanty low cost houses and rocky terrain. The only activity there is the multinational cement company, UNICEM. In addition, the rural area is separated from the transmitter (OkoiArikpo House) by dense vegetation and clusters of trees spread along the $24.1 \mathrm{~km}$ distance. The mean signal strength recorded was 87.7 dBm, signal penetration loss was $10.8 \mathrm{~dB}$, comparatively, these measured values deviated from the predicted values. This may be due to obstructions encountered by radio waves along the routes. Signal strength measurements for Akpabio, a rural enclave with signal path of $28.1 \mathrm{~km}$ from the transmitter station was $85.3 \mathrm{dBm}$, while signal penetration loss was $7.2 \mathrm{~dB}$. Results from Odukpani and Akamkpa were $83.1 \mathrm{dBm}$ and $87.5 \mathrm{dBm}$ for signal strength, while signal path loss recorded $6.7 \mathrm{dBm}$ and $7.6 \mathrm{dBm}$ respectively. The difference between the signal penetration loss in Calabar (urban) and the other four locations can be attributed to clusters of trees and dense vegetation located along the signal path.

\section{Conclusion}

From the study, wireless transmission shall be affected by signal attenuation in vicinity where the transmitting and 


\section{International Journal of Science and Research (IJSR) \\ ISSN (Online): 2319-7064 \\ Index Copernicus Value (2013): 6.14 | Impact Factor (2014): 5.611}

receiving radios are characterized by vegetation, thick forest and clusters of buildings. This may give rise deteriorated signal strength and path loss.

Additionally, fluctuations of radio wave propagation occur because of the movement of receiving terminal. Obstacles between the transmitter and receiving terminal, adverse environmental conditions invariably resulted to signal .fading, shadowing and path-loss. However, the study showed that propagation loss and signal strength levels were higher in locations with dense vegetation, high clusters of tree and sloppy topography. Studies have shown that diversity of radio wave signals caused by multi-path fading is sometimes more than $20 \mathrm{~dB}$ [1]. Earlier researchers had also highlighted that path loss increases significantly when propagated from outdoor-to-indoor under the frequency range of $455 \mathrm{MHz}-4.2 \mathrm{GHz}$ [2]. [6] conducted experiment on penetration loss for seven diverse houses in a suburban region at Chicago. From the results he presented, 16.3dB was favorable in a succinct comparison with the typical penetration loss. The value compared favorably with the average penetration loss of this study. Furthermore, [8] depicted that there is an observable decreases in penetration loss into suburban buildings as the frequency increases up to a range of 1-3 GHZ range. According to him, the signal loss at this frequency was between $7 \mathrm{~dB}$ to $8 \mathrm{~dB}$.

Prediction of the expected mean values for mobile received power is critical in planning of a mobile radio network coverage for such vicinity. These shall assist innovators, operators, etc., to determine the coverage area for Base stations and the minimum reuse distance for Base stations. The study shall find application in propagation prediction for sub urban and rural areas separated by vegetation and foliage.

\section{References}

[1] Andersen, J., Rappaport, T. \& Yoshida, S. (1995). Propagation measurements and models forwireless communicationchannels, IEEE Commun. Mag. 33: 4249.

[2] Andrew L. Martin (2007), VHF and Microwave Propagation Characteristics of Ducts, Andrew L. Martin, WirelessInstitute of Australia, January 17,XWE342007;

[3] Catrein, D., Reyer, M. \& Rick, T. (2007). Accelerating radiowave propagation predictionsby implementation on graphics hardware, Proc. IEEE Vehicular Technology Conference,Dublin, Ireland, pp. 510-514.

[4] Correia, L. M. (ed.) (2006). COST Action 273: Mobile Broadband Multimedia Networks, FinalReport, Academic Press.

[5] Damosso, E. (ed.) (1999). COST Action 231: Digital mobile radio towards future generation systems,Final Report, Office for Official Publications of the European Communities, Luxembourg.

[6] Erceg, V., Fortune, S. J., Ling, J., Rustako, J. \& Valenzuela, R. A. (1997). Comparison of acomputerbased propagation prediction tool with experimental data collected in urbanmicrocellular environments, IEEE J. Sel. Areas Commun. 15: 677-684.

[7] Erceg, V., Greenstein, L. J., Tjandra, S. Y., Parkoff, S. R., Gupta, A., Kulic, B., Julius, A. A. \&Bianchi, R.
(1999). An empirically based path loss model for wireless channels insuburban environments, IEEE J. Sel. Areas Commun. 17: 1205-1211.

[8] Hata, M. (1980). Empirical formula for propagation loss in land mobile radio services, IEEETrans. Veh. Technol. 29: 317-325.

[9] Ikegami, F., Takeuchi, T. \& Yoshida, S. (1991). Theoretical prediction of mean field strength forurban mobile radio, IEEE Trans. Antennas Propag. 39: 299302.

[10] Ikegami, F., Yoshida, S., Takeuchi, T. \&Umehira, M. (1984). Propagation factors controllingmean field strength on urban streets, IEEE Trans. Antennas.

[11]Ling, J. \& Moore, J. D. (1999). Radio propagation measurements and prediction usingthree-dimensional ray tracing in urban environments at $908 \mathrm{mhz}$ and 1.9 ghz, IEEETrans. Veh. Technol. 48: 931-946.

[12] Rajkumar, A., Naylor, B. F., Feisullin, F. \& Rogers, L. (1996). Predicting rf coverage in largeenvironments using ray-beam tracing and partitioning tree represented geometry,Wirel.Netw.2(2): 143-154.

[13] Rappaport, T. S. (1995). Wireless Communications: Principles and Practice, Prentice-Hall, Inc.Schaubach, K. R., IV, N. J. D.

[14] Rappaport, T. S. (1992). A ray tracing method for predictingpath loss and delay spread in microcellular environments, Proc. IEEE VehicularTechnology Conference, Vol. 2, pp. 932-935.

\section{Author Profile}

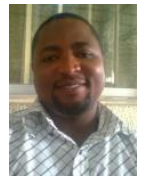

Okon, B. E. received the B.Sc. degree in Electronics and Computer Technology and M.Sc. degrees in Engineering Physics from the University of Calabar, Cross River State, Nigeria in 2007 and 2012, respectively. He is presently pursuing a Ph.D. degree in Engineering Physics in the same University. Within 2010-2015 he worked with the Ministry of Information and Communication Technology, a Ministry of the Cross River State Government.He is an acoustician, a Microsoft Certified Professional (MCP) and a Project Manager. 